Der Versuch, die Leiche für ein anatomisches Museum zu erwerben, misslang, ebenso die Erlaubaiss zur Section zu erlangen.

Die hartnäckige Nahrungsverweigerung des in einem brillanten Ernährungszustande $(2750 \mathrm{Grm}$.) geborenen Kindes ist jedenfalls auffallend. Interessant überhaupt würde wohl eine Zusammenstellung der bekannt gewordenen Fălle dieser Bildungsdefecte sein. Da selbst Barnum einen der Art defecten Menschen nie besessen hat, scheint mir frühester Tod das Schicksal dieser Wesen zu sein, denen möglicher Weise ein Defect im Centralnervensystem Nahrungsaufnahme und Fortleben unmöglich macht.

4.

\title{
Ueber das Wesen und die Behandlung einer Zahukrankheit.
}

\author{
Von Dr. med. von Platen, \\ pract. Arzt zu Vörde i. W.
}

Die Erkrankung, welche ich zu besprechen vorhabe, trägt den Charakter eines chronischen Leidens. Sie findet sich meist bei Lenten mit sogenannten schlechten Zähnen. Dennoch macht die Caries jedenfalls nicht das Wesen der Krankheit aus. Denn erstere kann schon seit 10 oder 15 Jahren in demselben Zustande verbarren. Nun beginnen zu einer Zeit mehr oder weniger heftige Schmerzen auf einer Seite. Ein Zabn ist der vorwiegend schmerzhafte. Die Schmerzen in ihm sind spannend, klopfend. Sie sind nicht gleichmässig intensiv. Namentlich ist ein schneller Wechsel der Temperatur, z. B. der Uebergang aus Winterskälte in die Hitze eines stark geheizten Zimmers, sehr geeignet, sle in heftiger Weise zu erregen. Die Zähne werden etwas lockerer dabei. Die betroffene Wange schwillt mässig an, schwillt beim Nachlass der Schmerzen vollständïg oder doch fast vollständig ab. Aeusserer Druck, die Berübrung des Zahnes mit dem Finger sind in diesen chrovischen Fällen nicht empfindlich. Empfindlicher ist das Hineinpressen des Zahnes in die Alveole beim Kauen, aus welchem Grunde dle Kranken diese Seite möglichst wenlg zu benutzen pflegen. In dieser Weise zieht sich das Leiden längere Zeit hin. Zeiten des Tages mit Nachlass oder gänzlichem Aufhören der Schmerzen wechseln mit schmerzhaften Perioden ab. In der Bettruhe wird zunächst der Schmerz etwas angefacht, bei längerer Ruhe vermindert er sich. Nun wird der Urheber des grössten Schmerzes entfernt. Die Extraction ist nicht sonderlich schmerzhaft, ausser bel den stark entzündeten Zăhnen (s. später). Die Schmerzen werden jetzt allmählich an Heftigkeit geringer, hören endlich für einige Zeit (Wochen bis Monate lang) ganz auf. Aber die Empfindlichkeit der Zâhne und hiermit die Neigung zum Recidiviren des Zahnschmerzes dauern an. Daher nehmen sich die Kranken oft ängstlich vor Erkältungen in Acht. Jedoch vergebens! Einmal wird durch eine Ursache, meist durch eine Erkältung, Schmerz in einem weiteren Zahne, auf derselben oder auf der anderen Seite hervorgerufen. Das Spiel beginnt jetzt von Neuem. So folgt denn eine Zahnextraction der anderen; aber eine Befreiung vom Debel ist nicht zu erreichen, bis vielleicht eine grosse Anzahl vou Zähnen entfernt ist. Und fast noch schlimmer, als das Uebel selbst, wirkt die Furcht vor demselben in den Zwischenzeiten, so dass in der That diesen Kranken der Genuss des Lebens in beträchtlicher Weise verkümmert werden kann.

Die Leute, die von dieser Krankheit befallen werden, haben das Körperwachsthum vollendet. 
Es kögnen nun Modificationen vorkommen. So sind zuweilen die Schmerzen in einem Zahne sebr heftig, sebr anhaltend; sie haben mehr das Auftreten, wie bei einem acuten Leiden. Oder es kann sich, bei bestimmten Personen namentlich, eine Parulis, eine Eiterung der Wurzelhaut, ausbilden.

Dies führt uns nun somit auf den Sitz des Leidens. Es ist wirklich eine Periostis dentis. Bei eingetretener Eiterung ist es sofort deutlich durch Usuren, selbst durch exostotische Vorragungen an der Wurzel des Zahnes. Ebenso wird es bei den Attaguen von acutem Charakter sehr überzeugend gezeigt. Der extrahirte Zahn ist an seiner Wurzel mit einer feinen, stark vascularisirten Haut überzogen. Aber auch die häufigsten, die ganz chronischen Fälle, Jassen etwas dahin Gehöriges erkennen, wenn auch nicht so sehr in die Augen fallend. Vergleicht man die Wurzel efnes solchen Zahnes mit der eines gesunden, so bemerkt man, dass die erstere nicht glatt ist, wie die gesunde. Sie ist nicht eigentlich rauh, aber doch uneben. Es finden sich Eindrücke, die thalartig, seicht um die ganze oder halbe Peripherie des Zahnes wohl herumlaufen. Oder es finden sich im Gegensatz auch leicht buckelartige Erhebungen. Zuweilen sehen die Eindrücke mehr gleichwie Löcherchen aus. Kurz, das Relief der sonst glatten Wurzel ist hier eln mannichfaltiges geworden. Man kann auch ein Stückehen verdickten Periostes an der Wurzel anhängend finden.

Die Periostitis erklärt nun die Zeichen des Schmerzes, den spannenden, klopfenden Charakter desselben, dann das Lockerwerden der Zähne, ihre Empfindlichkeit gegen Temperaturwechsel. Aber sie erklärt auch, - nnd das ist das Wichtigste, - das Recidiviren der Schmerzen. Denn die Periostitis beschränkt sich nicht anf elnen Zahn; sie erstreckt sich auch auf die übrigen derselben und selbst die der anderen Seite mehr oder weniger stark. Namentlich stnd es die anderen cariösen Zähne, we]che der Reihenfolge nach bis zu starker Schmerzhaftigkeit erkranken. Aeussere Reize sind für die Auslösung des Schmerzes nicht gleichgültig. Aber dass der äussere Reiz, der Jahre lang an solchen Zähnen ohne Beschwerde ertragen wurde, jetzt zur Wirkung gelangt, daran ist die Erkrankung des Periostes Schuld. Und dlese geht eine ganze Anzahl der Zähne, vielleicht oft alle Zähne des Patienten in stärkerem und in geringerem Grade zugleich an. Nun gilt es die Frage: Giebt es nicht gegen die Erkrankung des Periostes ein Mittel? Der Caries, welche in dem blutlosen Schmelze and dem blutlosen Dentin ihr Wesen treibt, kốnnen wir durch medicamentöse Behandlung wohl nicht abhelfen. Aber in Bezug auf das blutgefässhaltige Periost wäre Einiges zu versuchen. Die radicalste Cur ist allerdings die Extraction der ganz schlechten Zähne, die Plombirung der weniger schlechten, Anfertigung eines falschen Gebisses etc. Doch nicht jeder Mensch mag sich willig zu einem solchen Verfahren verstehen. Ich habe mir darum die Aufgabe gestellt, etwas zu suchen, welches die Periostitis zu beseitigen oder doch in Scbranken zu halten geeignet ist.

Chronische Periostitiden sind, wie wir wissen, überall sehr hartnäckig; ich lasse die specifischen hierbei weg. Aber eine Periostitis, welche man sich durch einen Stoss zugezogen hatte, deren gehörige Behandlung dann verabsänmt worden ist, so dass sie zu chronischer Verdickung geführt hat, kann sebr lange trotz aller Mittel fortbestehen. Doch die Schmerzhaftigkeit des Leidens nimmt mehr und mehr ab. Selbst Recidive sind leichter und weniger schmerzhaft, als das ursprüngliche Leiden. Es geht zur Vernarbung über. Nun ist dies bei den Periostitiden der Zähne wohl manchmal, aber in der Mehrzabl der Fälle nicht zu erreichen. Sie bewahren den entzündlich granulirenden Charakter. Ich kann dies nur mit dem Druck in der Alveole zusamménbringen. Auf alle Periostitiden wirkt ja Druck, wie wir wissen, sehr nachtheilig ein; leh erinnere an die Periostitiden an den Füssen. Zwar wird der Zahn durch einen heftigen Schmerzanfall etwas gelockert. Aber die Lockerung reicht nícht aus. Denn die Befestigung des Zahnes ist eine zu starke; die Einkeilung in die Alveole und die Verbindung mit dem Zabnfleisch, welches den Hals des Zahnes eng umspannt, halten und drängen den Zabn zurück. So besänftigt 
man auch den Schmerz momentan, wenn man den Zahn erfasst und thn hin und her bewegt, an ihm zieht, ohne dass die Linderung jedoch lange anhielte.

Ich kann gewiss meine Ansicht, den Forthestand der Periostitis durch den Druck in der Alveole zu erklären, nleht mit Beweisen belegen. Aber dass änssere Reize sie unterhalten soltten, dagegen spricht, dass selbst bei peinlicher Vermeidung jedes Reizes, wohl die Schmerzattaquen etwas nachlassen, aber nicht die Entzündung. Dann kann man dlesen Formen, so viel ich weiss, auch durch kein entzündungswidriges Mittel beikommen, wie anderen Periostitiden; Blutegel, Jodtinctur (auch bei anderen Formen des Zahnschmerzes oft nützlich) vermögen hier keinen dauernden Erfolg zu erzielen. Endlich möchte ich meine Ansicht folgern aus der günstigen Wirkung eines Mittels, welches ich in einer Belhe von Füllen erprobt habe, welches jedenfalls eine Lockerung des Zahnfleisches und der Zähne herbeiführt, das ist das Quecksilber.

Unter den Quecksilberpräparaten, in so weit sie anf die Schleimhaut des Mundes und deren Adnexa wirken, steht das Calomel so ziemlich obenan. Namentlich bei Erwachsenen treten schon nach wenigen Gaben leichte Formen der Mundentzündung auf. Ich wende es hier in kleinen Dosen an.

\section{Rec. Calomel. 0,05 \\ Sacch. 0,5.}

Um aber seine Wirkung im Munde möglichst stark zu machen, lasse ich es trocken anf die Zunge nehmen, lasse es dann nicht schlucken, sondern es bis zum voliständigen Zerfliessen im Munde behalten. Dann setzen sich die feinen Partikel des Calomel zwischen die Papillen der Zunge, und die Einwirkung wird eine viel länger dauernde. Ich lasse es ferner in nüchternem Zustande, etwa $\frac{1}{2}$ Stunde vor der Mahlzeit, nehmen. Bel heftlgen Zahnschmerzen gebe ich es dreimal täglich. In der Regel stellt sich nach kurzer Zeit mässige Diarrhoe eln. Haben am dritten Tage die Zabnschmerzen ziemlich nachgelassen, so setze ich im Laufe dieses Tages das Mittel aus. Ganz vorüber sind die Schmerzen Im Munde nicht. Der Zahnschmerz pflegt vorüber zn selo. Aber man empfindet ein unbestimmtes prickelndes und stechendes Gefühl im Klefer. Wahrscheinlich rührt es daher, weil die Zähne loser geworden sind. Sie müssen sich in den folgenden Tagen wieder befestigen, und dies Schmerzgefühl verliert sich ganz von selbst nach einiger Zeit.

Wenn der Zahnschmerz weniger heftig ist, oder recidivirt er nach einigen Wochen, so genügen oft $2-3$ Dosen, um ihn schnell zu beseitigen.

Nebenbei empfehle ich meinen Kranken an, sich durchaus nicht zu schonen, vielmehr viel das Freie aufzusuchen ohne Watte in den Ohren, ohne Kopftuch, ohne Halstuch. Ich wende dies Verfabren auch bei anderen Entzündungen an, z. B. bei leichten Anginen mit der bekannten trocknen Röthung der Schleimhaut. Dann halte ich den Hals nicht besonders warm, vielmehr vertausche ich den engeren Halskragen mit einem weiteren. Wenn die Entzündung einmal da ist, so sichert die Hyperämie vor einer weiteren Erkältung, - bis zu einer gewissen Grenze. Aber Wärme, verbunden mit dem Druck enger Kleidungsstücke, unterbält die Hyperämie. Nun treten solcherlet Zahnschmerzen in Zuständen auf, bei denen man von Anwendung des Calomel abstehen möchte, z. B. bel Schwangeren. Hier kann man die Elnreibung von grauer Salbe über die Wange versuchen. Ich habe hierüber noch zu wenig Erfahrung und stelle es weiteren Versuchen anhelm.

Ich habe schon gesagt, dass diese Art der Behandlung nur für die beschriebene Form der Periostitis passt. Für andere Anlässe des Zahnschmerzes, welche sich von Entzündungen der Pulpa herschreiben, Formen, wie sie namentlich im heranwacbsenden Alter vorkommen, sind gewiss entzündungswidrige Mittel mehr angebracht. Ich habe von der Application von Blutegeln auf das Zahnfleisch guten Erfolg geseben. Auch Pinselungen mit Jodtinctur auf das Zahnfleisch, welche empfohlen werden, wären hierbei wobl anzuwenden. 